Florida International University FIU Digital Commons

10-30-2014

\title{
New Bivariate Lifetime Distributions Based on Bath-Tub Shaped Failure Rate
}

Mengying Li

mli032@fiu.edu

DOI: $10.25148 /$ etd.FI14110710

Follow this and additional works at: https://digitalcommons.fiu.edu/etd

Part of the Statistics and Probability Commons

\section{Recommended Citation}

Li, Mengying, "New Bivariate Lifetime Distributions Based on Bath-Tub Shaped Failure Rate" (2014). FIU Electronic Theses and Dissertations. 1583.

https://digitalcommons.fiu.edu/etd/1583

This work is brought to you for free and open access by the University Graduate School at FIU Digital Commons. It has been accepted for inclusion in FIU Electronic Theses and Dissertations by an authorized administrator of FIU Digital Commons. For more information, please contact dcc@fiu.edu. 
FLORIDA INTERNATIONAL UNIVERSITY

Miami, Florida

NEW BIVARIATE LIFETIME DISTRIBUTIONS

BASED ON BATH-TUB SHAPED FAILURE RATE

A thesis submitted in partial fulfillment of the

requirements for the degree of

MASTER OF SCIENCE

in

STATISTICS

by

Mengying Li

2014 
To: Interim Dean Michael R. Heithaus

College of Arts and Sciences

This thesis, written by Mengying Li, and entitled New Bivariate Lifetime Distributions Based on Bath-tub Shaped Failure Rate, having been approved in respect to style and intellectual content, is referred to you for judgment.

We have read this thesis and recommend that it be approved.

Florence George

Zhenmin Chen

Hassan Zahedi, Major Professor

Date of Defense: October 30, 2014

The thesis of Mengying Li is approved.

Interim Dean Michael R. Heithaus

College of Arts and Sciences

Dean Lakshmi N. Reddi

University Graduate School

Florida International University, 2014 


\section{ACKNOWLEDGMENTS}

First of all, I would like to express my sincere thanks to my major professor, Dr. Hassan Zahedi for his patient guidance, enthusiasm, encouragement and friendship throughout this whole study. I couldn’t finish my research without his great support.

I would also like to thank the members of my committee, Dr. Florence George and Dr. Zhenmin Chen for their time, valuable advice and great encouragement. In addition, I would like to thank the Department of Mathematics and Statistics, all the professors who supported and encouraged me throughout my life in Florida International University.

Finally, I will express my appreciation to my parents and friends for their encouragement and support. 


\begin{abstract}
OF THE THESIS
NEW BIVARIATE LIFETIME DISTRIBUTIONS

BASED ON BATH-TUB SHAPED FAILURE RATE
\end{abstract}

by

Mengying Li

Florida International University, 2014

Miami, Florida

Professor Hassan Zahedi, Major Professor

A class of lifetime distributions which has received considerable attention in modelling and analysis of lifetime data is the class of lifetime distributions with bath-tub shaped failure rate functions because of their extensive applications. The purpose of this thesis was to introduce a new class of bivariate lifetime distributions with bath-tub shaped failure rates (BTFRFs). In this research, first we reviewed univariate lifetime distributions with bath-tub shaped failure rates, and several multivariate extensions of a univariate failure rate function. Then we introduced a new class of bivariate distributions with bath-tub shaped failure rates (hazard gradients). Specifically, the new class of bivariate lifetime distributions were developed using the method of Morgensterns method of defining bivariate class of distributions with given marginals. The computer simulations and numerical computations were used to investigate the properties of these distributions.

Keywords: Failure Rate, Hazard Gradient, Lifetime Distribution, Bivariate Bath-tub Shaped Failure Rate. 


\section{TABLE OF CONTENTS}

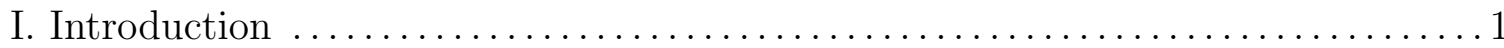

II. Review of the Definitions and Theorems $\ldots \ldots \ldots \ldots \ldots \ldots \ldots \ldots \ldots \ldots \ldots \ldots$

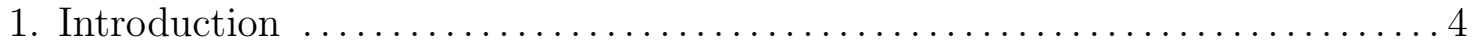

2. Univariate Lifetime Distribution with BTFRF $\ldots \ldots \ldots \ldots \ldots \ldots \ldots \ldots$

III. New Class of Bivariate Lifetime Distributions with BTFRFs $\ldots \ldots \ldots \ldots \ldots . .8$

1. Multivariate Extensions of Univariate Failure Rate Function $\ldots \ldots \ldots \ldots \ldots 8$

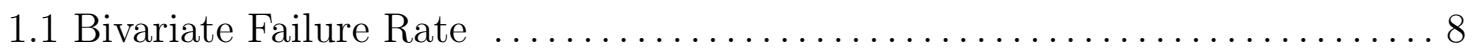

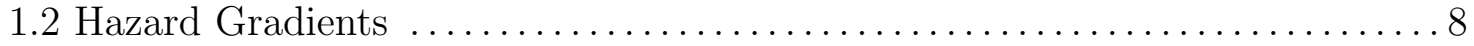

2. New class of Bivariate Lifetime Distributions with BTFRFs . ............ 11

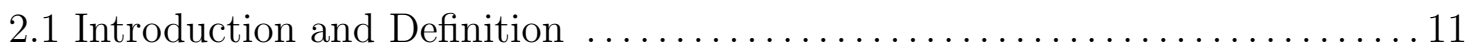

2.2 Some Properties of the New Bivariate Lifetime Distributions with BTFRFs 14

IV. Review of Some Classes of Bivariate Distributions with Given Marginals ... 18

1. Morgenstern's Class of Bivariate Distributions ..................... 18

2. Farlie's Class of Bivariate Distributions .......................... 19

3. Plackett's Class of Bivariate Distributions ........................20

V. New Class of Bivariate Distribution Based on Weibull Mixture Distribution ..21

1. Review of Univariate Mixture Weibull Distribution with BTFRF .........21

2. New Class of Bivariate Distribution with Mixture Weibull as Marginals ... 22

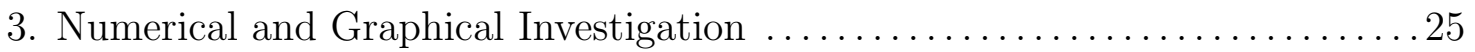

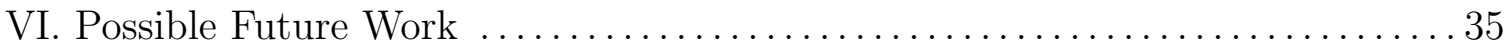

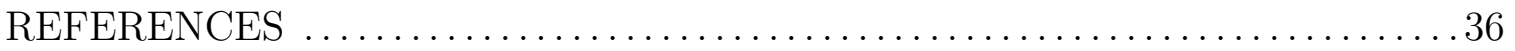




\section{LIST OF FIGURES}

FIGURE

PAGE

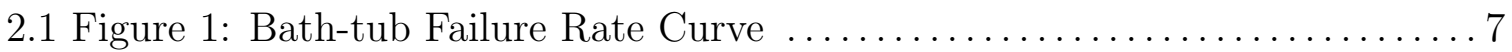

5.1 Figure 2: $H(x, y)$ when $\beta_{1}=0.3, \beta_{2}=1.5$, and $\alpha=0.5 \ldots \ldots \ldots \ldots \ldots \ldots 27$

5.2 Figure 3: $r_{x}(x, y)$ when $\beta_{1}=0.3, \beta_{2}=1.5, \alpha=0 \ldots \ldots \ldots \ldots \ldots \ldots \ldots$

5.3 Figure 4: $r_{x}(x, y)$ when $\beta_{1}=0.5, \beta_{2}=4, \alpha=0 \ldots \ldots \ldots \ldots \ldots \ldots \ldots \ldots \ldots$

5.4 Figure 5: $r_{x}(x, y)$ when $\beta_{1}=0.5, \beta_{2}=4, \alpha=0.5 \ldots \ldots \ldots \ldots \ldots \ldots \ldots \ldots 32$ 


\section{Introduction}

In many applications in reliability, failure rate function is used for modelling lifetime data. The aging process can be effectively modelled and described by the shape of the failure rate functions. Large failure rates imply more chance to fail at that time point, and small failure rates imply less change to fail at that time point. A natural progress of aging is reflected by an increasing failure rate, and an improvement over time is reflected by a decreasing failure rate. If neither wear out or improvement effect is shown, then the failure rate is flat, which implies a constant failure rate. In many applications in reliability, the failure rate function can be bath-tub shaped. A Bath-tub Shaped Failure Rate Function(BTFRF), occurs when the failure rate of an item decreases at the beginning, then stays constant, and finally increases as an item ages. That is, the item improves initially, remains stable for some time and then degrades as time passes (wear-out effect), which occurs in the manufacturing products. Often the manufacturing process, design, or component defects cause early failure, which is commonly called the "burn-in" period. Once items pass through this early critical part of their lifetimes, they have a fairly constant failure rate for some time. After this period of time, the change point $a$, the item continues to age and the failure rate increases reflecting in a "wear out" period. These three phases can be effectively modelled by a survival distribution which has a bath-tub shaped failure rate function, usually called bath-tub shaped model. There are many areas of applications, such as reliability analysis in engineering, survival analysis in biostatistics, where these results can be used. 
A large number of studies have been done to characterize class of lifetime distributions which exhibit a bath-tub shaped failure rate function. For example, Xie and Lai (1995) proposed an additive Weibull model which has a BTFRF, Mudholkar et al. (1995) introduced the exponential Weibull distribution which has a BTFRF, and Ghitany (2004) examined a beta distribution which has a BTFRF. All the studies mentioned were univariate lifetime distributions with a BTFRF. Therefore, it is of interest to extend this concept to multivariate lifetimes, since in reliability, systems are usually composed of a plurality of components rather than a single component. In the present research, a new generalization was made from a univariate lifetime distribution with a BTFRF to a bivariate class of lifetime distribution with BTFRFs.

In this study, Chapter 1 includes an introduction, a brief review of recent studies of this topic, and an introduction of the contents of this study. Chapter 2 contains a review of basic concepts and theorems in survival analysis. Especially, the review of the univariate lifetime distribution with a BTFRF. Chapter 3 introduces a new class of bivariate lifetime distributions with BTFRFs. In this chapter, two methods of the multivariate extensions of univariate failure rate function are reviewed, among which the notion of hazard gradient is used in this research. Afterwards, the definition and general form of bivariate lifetime distributions with BTFRFs are derived. Chapter 4 reviews several ways of generating a bivariate distribution with given marginals. One of the methods (Morgenstern's method) is chosen for this research. Using Morgenstern's method, a new class of bivariate distribution with BTFRFs is introduced. Chapter 5 contains the main results of the study. It contains 
an example of this new class of bivariate lifetime distributions, using mixture of Weibull distributions as its marginals. Furthermore, numerical and graphical results are used to investigate the properties of this new class of bivariate distributions. Chapter 6 contains some suggestions for future works that I plan to investigate. 


\section{Review of the Definitions and Theorems}

\section{Introduction}

In reliability analysis, various distributions can be used to model lifetime data. Various ways of modelling lifetime data are available, derived from survival function or failure rate function. Survival function is the probability that an item is functioning beyond a specific time. The survival function is defined by:

$$
S(t)=P[T>t]
$$

A survival function is also known as the reliability function in terms of Cumulative Distribution Function (CDF) is given by:

$$
S(t)=1-F(t)=\overline{F(t)}
$$

where $F$ is the CDF of the unit.

Failure rate function (or hazard function) is another important method in modelling lifetime data. Failure rate function associates the risk to a specific time $t$. One can observe the change of risk with time by plotting the failure rate function. When $T$ is continuous, the failure rate function is defined as:

$$
r(t)=\frac{f(t)}{S(t)}
$$

Note that:

$$
r(t)=\lim _{\delta \rightarrow 0} \frac{P[T+\delta \leq t \mid T>t]}{\delta}
$$

The failure rate represents that, given that an item has survived up to time $t$, the rate (or chance) that it will fail instantly after time $t$. Failure rate is an important 
concept in insurance, finance, commerce, and engineering, and it is fundamental in analysis of lifetimes in a wide variety of applications.

\section{Univariate Lifetime Distribution with BTFRF}

The shape of a failure rate function can be usually generalized into four categories: the first one is monotonic Increasing Failure Rate (IFR), where the shape of the failure rate is monotonic increasing. The second category is monotonic Decreasing Failure Rate (DFR), where the shape of the failure rate is monotonic decreasing. The third one is the Bath-Tub shaped failure rate (BT), where the shape of the failure rate is decreasing before time $t_{1}$, stays constant within $t_{1}$ and $t_{2}$, and finally increasing after point $t_{2}$. The last one is the Upside-down Bath-Tub shaped failure rate(UBT), where the shape of the failure rate is increasing before time $t_{1}$, stays constant within $t_{1}$ and $t_{2}$, and finally decreasing after time $t_{2}$. For the third one and the forth one, $t_{1}$ could be equal to $t_{2}$, which results $\mathrm{U}$-shaped or $\mathrm{V}$-shaped failure rates and inverse U-shaped or V shaped failure rates.

In reliability, lifetime distribution with bath-tub shaped failure rate (BT) has been used frequently. Typically, the period that the shape of the failure rate is decreasing is called a "burn-in" period, the period that the failure rate stays constant is called a "constant period", and the period that the shape of the failure rate is decreasing is called a "wear-out" period. If in some special case $t_{1}$ equals $t_{2}$, then BT only has a "burn-in" period and a "wear-out" period, which is one generalization of a bath-tub shaped failure rate.

Definition Let $r(t)$ be the failure rate function of a lifetime distribution $F$, we have 
the following classes of lifetime distributions:

i. IFR (Increasing Failure Rate) if the failure rate function is monotonic increasing. ii. DFR (Decreasing Failure Rate) if the failure rate function is monotonic decreasing. iii. BT (Bath-tub Shaped Failure Rate) if there exists a $t_{1}$ such that the failure rate function is nonincreasing when $0<t<t_{1}$, remaining constant when $t_{1} \leq t<t_{2}$, and nondecreasing when $t \geq t_{2}$.

iv. UBT (Upside-down Bath-tub Shaped Failure Rate) if there exists a $t_{1}$ such that the failure rate function is nondecreasing when $0<t<t_{1}$, remaining constant when $t_{1} \leq t<t_{2}$, and nonincreasing when $t \geq t_{2}$.

The bath-tub shaped survival functions have been studied extensively. See E.Halley(1693), R.E Barlow and F.Proschan(1975), B.L. Amstadter(1977), and F.Jensen(1989) for examples. Some of the studies focus on how to characterize the increasing and decreasing trend of the failure rate, such as Ronald E. Glaser (1980). He presents a sufficient condition for a lifetime distribution to result in a bath-tub shaped failure rate function. Ebeling (1997) expresses bathtub curve as a composite of several failure distributions. In conclusion, a large number of bath-tub models have been developed, and typical methods to establish a bathtub curve model involves seeking an appropriate model, modifying the model by introducing additional parameters, and transforming the data to achieve compatibility with the well developed and understood model. 


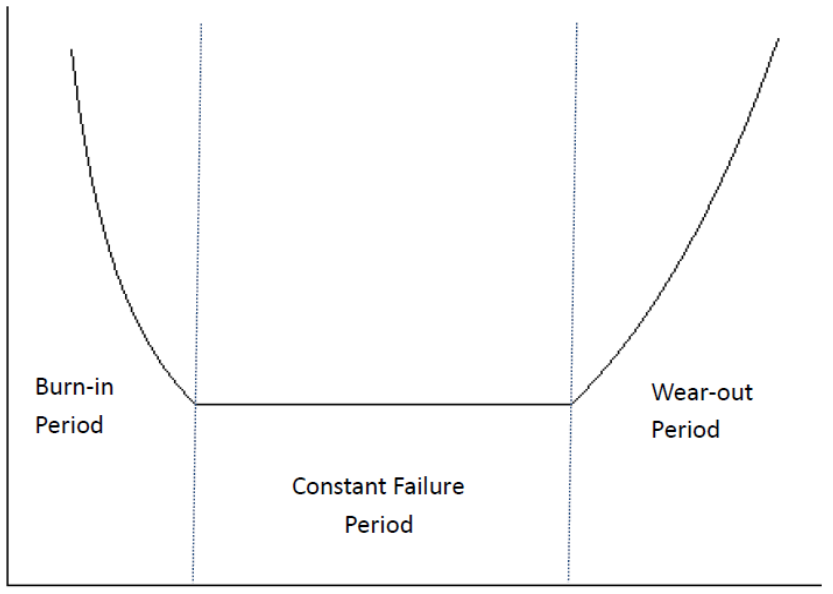

Figure 1: Bath-tub Failure Rate Curve 


\section{New Class of Bivariate Lifetime Distributions with BTFRFs \\ 1. Multivariate Extensions of Univariate Failure Rate Function}

\subsection{Bivariate Failure Rate}

The first bivariate extension of a univariate failure rate function definition is given in Basu (1971) and is as follows. Given an absolutely continuous bivariate distribution function $H(x, y)$ with density function $f(x, y)$, the bivariate failure rate is defined as:

$$
r(x, y)=\frac{f(x, y)}{P(X>x, Y>y)}=\frac{f(x, y)}{\bar{H}(x, y)}
$$

Since $P(X>x, Y>y)=1+F(x, y)-F(x, \infty)-F(\infty, y)$, it is very easy to check

that when $X$ and $Y$ are independent, $r(x, y)=r(x) r(y)$, where $r(x)$ and $r(y)$ are the corresponding univariate failure rates. Furthermore, using this definition given by equation (5), he showed that no absolutely continuous bivariate distribution with constant failure rate exists unless in the special case when $X$ and $Y$ are independently distributed. However, this definition is not used for this research, since the bivariate failure rate(s) given by equation (5) does not have a bivariate loss of memory property, and it does not uniquely characterize the joint distribution function of $X$ and $Y$.

\subsection{Hazard Gradients}

The extension to bivariate failure rates, called the hazard gradients defined by Johnson and Kotz (1975), Barlow and Proschan (1975), and Marshall (1975). In the univariate case, the failure rate function is a measurement of the force or rate of mortality. When it comes to bivariate and multivariate cases, the rate of mortality depends on which variable is changed. As a result, the hazard gradient is an suitable 
function for modelling the multivariate lifetime data.

Definition (Johnson and Kotz, 1975) The joint multivariate hazard gradients of $m$ absolutely continuous random variables $X_{1}, \ldots X_{m}$ are defined as the vector:

$$
\mathbf{r}_{\mathbf{x}}(\mathbf{x})=\left(-\frac{\partial}{\partial x_{1}}, \ldots,-\frac{\partial}{\partial x_{m}}\right) \log \overline{H_{x}}(x)
$$

where $H_{x}(\mathbf{x})=P\left(X_{i}>x_{i}, i=1, \ldots, m\right)$. For convenience we will write

$$
r_{j}(\mathbf{x})=-\frac{\partial}{\partial x_{j}} \log \overline{H_{x}}(x), j=1, \ldots, m
$$

where $H_{x}(\mathbf{x})$ is the multivariate life distribution. In this research, we restrict our study to the bivariate case. The definition for the bivariate case can be written as:

Definition The bivariate hazard gradients of two absolutely continuous random variables $X$ and $Y$ are:

$$
r_{x}(x, y)=-\frac{\partial}{\partial x} \log \bar{H}(x, y)=\frac{-\frac{\partial}{\partial x} \bar{H}(x, y)}{\bar{H}(x, y)}
$$

and

$$
r_{y}(x, y)=-\frac{\partial}{\partial y} \log \bar{H}(x, y)=\frac{-\frac{\partial}{\partial y} \bar{H}(x, y)}{\bar{H}(x, y)}
$$

where $\bar{H}(x, y)$ is the joint survival function for $X$ and $Y$. According to the definition, if for each $x$ and $y, r_{x}(x, y)$ and $r_{y}(x, y)$ are increasing (decreasing) functions of the corresponding variables, then the corresponding bivariate distribution is called a bivariate IHR $(\mathrm{DHR}) .(\mathrm{IHR}=\mathrm{IFR}$ and $\mathrm{DHR}=\mathrm{DFR})$

In the univariate case, it is easy to check that there is a one-to-one relationship between failure rate function and the survival function. That is,

$$
\overline{H(x)}=e^{-\int_{0}^{x} r(z) d z}, t \geq 0
$$


The above equation gives the survival function $\overline{H(x)}$ in terms of the failure rate function $r(x)$ and hence there is a one to one correspondence between a failure rate and its survival function.

For the multivariate case, this relationship holds as well. Note that, the random variables are $X_{1}, X_{2}, \ldots, X_{m}$, and the joint survival function $\overline{H(x)}$ is:

$$
\overline{H(\mathbf{x})}=P\left(X_{1}>x_{1}, X_{2}>x_{2}, \ldots, X_{m}>x_{m}\right)
$$

defined on the $S=\{\mathbf{x}: \bar{H}(\mathbf{t})>0\}$. Marshall (1975) defines a "hazard function" corresponding to the survival function $\overline{H(\mathbf{x})}$ as:

$$
R(\mathbf{x})=-\log \overline{H(\mathbf{x})}
$$

The relationship between hazard function and the hazard gradients is given by:

$$
R(\mathbf{x})=\int_{C} r(\mathbf{z}) d \mathbf{z}
$$

where the integral is a line integral and $\mathrm{C}$ is any sufficient continuous path that begins at $\mathbf{0}=(0,0, \ldots, 0)$ and ends at $\mathbf{X}, R(\mathbf{x})$ is the hazard function, and $r(\mathbf{x})$ is the hazard gradients for $\mathbf{X}$. It is easy to check that its survival function $\bar{H}(\mathbf{x})$ can be obtained by:

$$
\bar{H}(\mathbf{x})=e^{-\int_{C} r(\mathbf{z}) d \mathbf{z}}
$$

From the above equation, one can choose a path of particular interest from $\mathbf{0}=$ $(0,0, \ldots, 0)$ to $\left(x_{1}, x_{2}, \ldots, x_{m}\right)$, therefore, the hazard function and the corresponding 
survival function $\bar{H}(\mathbf{x})$ can be obtained as follows:

$$
\begin{array}{r}
R(\mathbf{x})=\int_{0}^{x_{1}} r_{1}\left(z_{1}, 0, \ldots, 0\right) d z_{1}+\int_{0}^{x_{2}} r_{2}\left(x_{1}, z_{2}, \ldots, 0\right) d z_{2}+\ldots \\
\ldots+\int_{0}^{x_{m}} r_{m}\left(x_{1}, x_{2}, \ldots, x_{m-1}, z_{m}\right) d z_{m}
\end{array}
$$

and

$$
\bar{H}(\mathbf{x})=e^{-\left\{\int_{0}^{x_{1}} r_{1}\left(z_{1}, 0, \ldots, 0\right) d z_{1}+\int_{0}^{x_{2}} r_{2}\left(x_{1}, z_{2}, \ldots, 0\right) d z_{2}+\ldots+\int_{0}^{x_{m}} r_{m}\left(x_{1}, x_{2}, \ldots, x_{m-1}, z_{m}\right) d z_{m}\right\}}
$$

where $m=2$, this reduces to the bivariate case. That is, let $r_{x}(x, y)$ and $r_{y}(x, y)$ be the hazard gradients corresponding to $\bar{H}(x, y)$, then the joint survival function is given by:

$$
\bar{H}(x, y)=e^{-\left\{\int_{0}^{x} r_{x}\left(z_{1}, 0\right) d z_{1}+\int_{0}^{y} r_{y}\left(x, z_{2}\right) d z_{2}\right\}}
$$

That is, similar to the univariate case, the hazard gradient characterizes the joint survival function $\bar{H}(x, y)$.

\section{New Class of Bivariate Lifetime Distributions with BTFRFs}

\subsection{Introduction and Definition}

In the present research, hazard gradients are used to generate a new class of bivariate lifetime distributions, which extends the BTFRF from the univariate case to the bivariate case. First, we introduce some existing definitions of bivariate classes of survival functions.

Definition I Let $r_{x}(x, y)$ and $r_{y}(x, y)$ be hazard gradients of a bivariate lifetime $(X, Y)$ with corresponding joint $\mathrm{CDF}, H(x, y)$, and joint survival function $\bar{H}(x, y)$. We say the: 
i. IFR (Increasing Failure Rate), if $r_{x}(x, y)$ is monotonic increasing with respect to $x$, for all $y$, and $r_{y}(x, y)$ is monotonic increasing with respect to $y$, for all $x$.

ii. DFR (Decreasing Failure Rate) if $r_{x}(x, y)$ is monotonic decreasing with respect to $x$, for all $y$, and $r_{y}(x, y)$ is monotonic decreasing with respect to $y$, for all $x$.

New definitions of bivariate classes of survival functions are as follows:

\section{Definition II}

i. The bivariate joint survival function $\bar{H}(x, y)$ has a BT (Bath-tub Shaped Failure Rates) if:

a) $r_{x}(x, y)$ is bath-tub shaped with respect to $x$ in all $y$.

b) $r_{y}(x, y)$ is bath-tub shaped with respect to $y$ in all $x$.

ii. The bivariate joint survival function $\bar{H}(x, y)$ has a UBT (Upside-down Bath-tub Shaped Failure Rates) if:

a) $r_{x}(x, y)$ is upside-down bath-tub shaped with respect to $x$ in all $y$.

b) $r_{y}(x, y)$ is upside-down bath-tub shaped with respect to $y$ in all $x$.

That is, if $H(x, y)$ and $\bar{H}(x, y)$ denote the joint CDF and joint survival function for lifetimes $X$ and $Y$, and $r_{x}(x, y)$ and $r_{y}(x, y)$ as the corresponding hazard gradients, then according to definition II, $H(x, y)$ has BTFRFs if:

$$
r_{x}(x, y)= \begin{cases}g_{1}(x, y) & 0<x<t_{1}, \forall y \in R_{+} \\ c_{1}(y) & t_{1} \leq x<t_{2}, \forall y \in R_{+} \\ g_{2}(x, y) & x \geq t_{2}, \forall y \in R_{+}\end{cases}
$$

where, $g_{1}(x, y)$ is nonincreasing with respect to $x$ where $0<x<t_{1}, \forall y \in R_{+} ; c_{1}(y)$ 
is only a function of $y$ where $t_{1} \leq x<t_{2}, \forall y \in R_{+}$; and $g_{2}(x, y)$ is a nondecreasing function of $x$ where $x \geq t_{2}, \forall y \in R_{+}$. And,

$$
r_{y}(x, y)= \begin{cases}g_{3}(x, y) & 0<y<s_{1}, \forall x \in R_{+} \\ c_{2}(x) & s_{1} \leq y<s_{2}, \forall x \in R_{+} \\ g_{4}(x, y) & y \geq s_{2}, \forall x \in R_{+}\end{cases}
$$

where $g_{3}(x, y)$ is a nonincreasing function of $y$ where $0<y<s_{1}, \forall x \in R_{+} ; c_{2}(x)$ is only a function of $x$ where $s_{1} \leq y<s_{2}, \forall x \in R_{+}$, and $g_{4}(x, y)$ is nondecreasing in $y$ where $y \geq s_{2}, \forall x \in R_{+}$.

Note equation (18) and (19) imply that $r_{x}(x, y)$ is bath-tub shaped with respect to $x, \forall y \in R_{+}$; and $r_{y}(x, y)$ is bath-tub shaped with respect to $y, \forall x \in R_{+}$. If any bivariate lifetime distribution $H(x, y)$ has hazard gradients $r_{x}(x, y)$ and $r_{y}(x, y)$ that satisfy (18) and (19), we say this bivariate lifetime distribution has BTFRFs.

For a special case where $t_{1}=t_{2}$ and $s_{1}=s_{2}$, the hazard gradient only has a "burn-in" period and a "wear-out" period. Though the definition of the bivariate BTFRFs is very similar to the definition of the univariate case, the constant period parts for hazard gradients are not strict constants in the bivariate case. That means, $c_{1}(y)$ is an function of $Y$ only, and does not change with the change of $X$, and $c_{2}(x)$ is an function of $X$ only, and does not change with the change of $Y$. For a special case with strict constant failure rates, one can define $c_{1}(y)$ and $c_{2}(x)$ as two strict constants instead of two functions. 


\subsection{Some Properties of the New Bivariate Lifetime Distributions with BT-}

\section{FRFs}

In section 3.1.2, we discussed the relationship between hazard gradients $r_{x}(x, y)$ and $r_{y}(x, y)$ and the survival functions.

Theorem I If survival function $\bar{H}(x, y)$ has BTFRFs with hazard gradients given by (18) and (19), the joint survival function is given by:

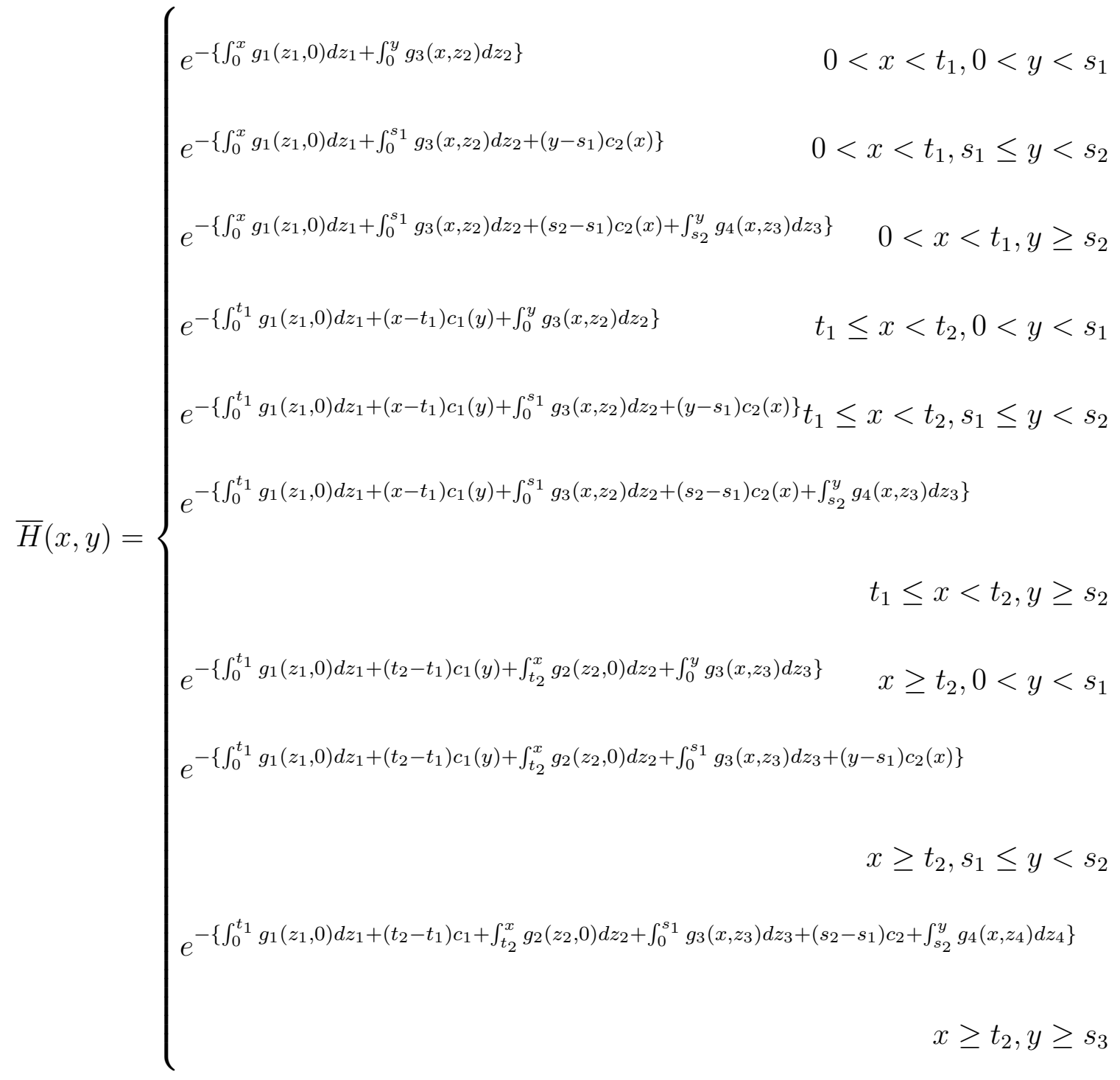

Proof: it follows by using the result that $\bar{H}(x, y)=e^{-\left\{\int_{0}^{x} r_{x}\left(z_{1}, 0\right) d z_{1}+\int_{0}^{y} r_{y}\left(x, z_{2}\right) d z_{2}\right\}}$ for a 
possible region for values of $(x, y)$.

Theorem II If $\bar{H}(x, y)$ has BTFRFs, then its univariate marginals also have BTFRFs, but converse need not to be ture.

Proof: proof is straight forward by letting $y=0$ in (18) and $x=0$ in (19).

As the equation (20) shows, where $t_{1}<t_{2}$, the computations become extremely difficult. In this research, we consider the special case where $t_{1}=t_{2}$ and $s_{1}=s_{2}$, which indicate that hazard gradients are U-shaped or V-shaped BTFRFs without the constant failure rate periods. In this case, the hazard gradients in (18) and (19) reduces to:

$$
r_{x}(x, y)= \begin{cases}g_{1}(x, y) & 0<x<t_{1}, \forall y \in R_{+} \\ g_{2}(x, y) & x \geq t_{1}, \forall y \in R_{+}\end{cases}
$$

where, $g_{1}(x, y)$ is nonincreasing with respect to $x$ where $0<x<t_{1}, \forall y \in R_{+}$, and $g_{2}(x, y)$ is nondecreasing of $x$ where $x \geq t_{1}, \forall y \in R_{+}$. And,

$$
r_{y}(x, y)= \begin{cases}g_{3}(x, y) & 0<y<s_{1}, \forall x \in R_{+} \\ g_{4}(x, y) & y \geq s_{1}, \forall x \in R_{+}\end{cases}
$$

where $g_{3}(x, y)$ is nonincreasing of $y$ where $0<y<s_{1}, \forall x \in R_{+}$, and $g_{4}(x, y)$ is nondecreasing of $y$ where $y \geq s_{1}, \forall x \in R_{+}$. In this case, the survival function $\bar{H}(x, y)$ 
is given by:

$$
\bar{H}(x, y)=\left\{\begin{array}{lr}
e^{-\left\{\int_{0}^{x} g_{1}\left(z_{1}, 0\right) d z_{1}+\int_{0}^{y} g_{3}\left(x, z_{2}\right) d z_{2}\right\}} & 0<x<t_{1}, 0<y<s_{1} \\
e^{-\left\{\int_{0}^{x} g_{1}\left(z_{1}, 0\right) d z_{1}+\int_{0}^{s_{1}} g_{3}\left(x, z_{2}\right) d z_{2}+\int_{s_{1}}^{y} g_{4}\left(x, z_{3}\right) d z_{3}\right\}} & 0<x<t_{1}, y \geq s_{1} \\
e^{-\left\{\int_{0}^{t_{1}} g_{1}\left(z_{1}, 0\right) d z_{1}+\int_{t_{1}}^{x} g_{2}\left(z_{2}, 0\right) d z_{2}+\int_{0}^{y} g_{3}\left(x, z_{3}\right) d z_{3}\right\}} & x \geq t_{1}, 0<y<s_{1} \\
e^{-\left\{\int_{0}^{t_{1}} g_{1}\left(z_{1}, 0\right) d z_{1}+\int_{t_{1}}^{x} g_{2}\left(z_{2}, 0\right) d z_{2}+\int_{0}^{s_{1}} g_{3}\left(x, z_{3}\right) d z_{3}+\int_{s_{1}}^{y} g_{4}\left(x, z_{4}\right) d z_{4}\right\}} x \geq t_{1}, y \geq s_{1}
\end{array}\right.
$$

The above equation is the general expression of the survival function for the bivariate lifetime distribution with a V-shaped or U-shaped BTFRFs. Theoretically, with the equation (20) and (23), one can obtain the survival function of the bivariate lifetime $(X, Y)$, if the bath-tub shaped hazard gradients are given. The original design of the study was to find the joint survival function if the hazard gradients are set to be of some special cases. That is, one could choose specific $g_{1}(x, y), g_{2}(x, y), g_{3}(x, y)$, and $g_{4}(x, y)$, afterwards, $\bar{H}(x, y)$ could be obtained using (20) or (23). However, the approach that using (20) or (23) requires very complex computations, and could lead to a result that is too "random" and is beyond the scope of this research. Hence, we use the following approach.

Since the univariate marginals need to be BTFRFs, we focus on bivariate distribution for which their univariate marginals are BTFRFs. That is, a permissible bivariate distribution with given marginals is generated first, then investigation is needed to see if this bivariate distribution satisfies the definition of the bivariate lifetime distribution with BTFRFs. If the new class of bivariate lifetime distributions 
has hazard gradients that are bath-tub shaped (See Definition II), then it is bivariate BTFRF survival functions. Certain questions need to be answered: Are the hazard gradients bath-tub shaped? Is this new class of bivariate lifetime distribution a permissible extension of the univariate case? What happens if $X$ and $Y$ are independent or correlated? Fortunately, various approaches are available to generate a proper bivariate distribution with given marginals, which will be discussed in the next chapter. 


\section{Review of Some Classes of Bivariate Distributions with Given Marginals}

In statistics, it is of interest to develop some multivariate distributions with some given properties. For example, consider a machinery component that consists part A and Part B. Damage of the component may result from three sources: Damage of $\mathrm{A}$, damage of $\mathrm{B}$, or jointly damage of $\mathrm{A}$ and $\mathrm{B}$. Occasionally, the component may be affect simultaneously from both sources. If we let $X$ represent the working condition of part $\mathrm{A}$ and $Y$ represent the working condition of part $\mathrm{B}$, then $(X, Y)$ is a bivariate random variable. Since the performance of Part A and Part B are known individually, it is of interest to explore the performance of $(X, Y)$, when we know the performance of $X$ and $Y$ individually.

There are numerous studies about the ways to generate multivariate distribution with given properties, especially the studies on the method of defining bivariate distributions using given marginals. For example, using Nataf's (1962) method of translation, it is possible to construct families of bivariate distribution whose marginals, $F(x)$ and $G(y)$, are given arbitrary. Morgenstern (1956) introduced a system which was extended by Farlie (1960) to generate a bivariate distribution with given marginals. Statistician R.L. Plackett (1965) introduced a method of constructing a one parameter class of bivariate distribution with given marginals. All methods could give a permissible class of bivariate distribution with some given desired properties.

\section{Morgenstern's Class of Bivariate Distributions}

Morgenstern(1956) introduced a system of bivariate distribution with given 
marginals. The Morgenstern's method is the one that we use in this research. Let $F(x)$ and $G(y)$ be two given univariate CDFs, Morgenstern's class of bivariate distribution with marginals $F(x)$ and $G(y)$ is given by:

$$
H(x, y)=F(x) G(y)[1+\alpha(1-F(x))(1-G(y))]
$$

The class of bivariate distributions would have $F(x)$ and $G(y)$ as its univariate marginals where parameter $\alpha$ is a measure of dependence between $X$ and $Y$. For $H(x, y)$ to be a proper continuous bivariate distribution function, the following conditions need to be satisfied:

i. $H(x, y)$ is a differentiable monotonic increasing function of both $\mathrm{x}$ and $\mathrm{y}$.

ii. $0 \leq H(x, y) \leq 1$ for all $\mathrm{x}$ and $\mathrm{y}$.

iii. $\lim _{x, y \rightarrow+\infty} H(x, y)=1$.

iv. $H(x, y)$ is non-decreasing with respect to $x$ and $y$.

v. $H(x, y)$ must satisfy rectangular inequality.

For $H(x, y)$ to have marginal distribution functions $F(x)$ and $G(y), \lim _{x \rightarrow \infty} H(x, y)=$ $G(y)$ and $\lim _{y \rightarrow \infty} H(x, y)=F(x)$ should be further satisfied.

\section{Farlie's Class of Bivariate Distributions}

Farlie(1960) extended Morgenstern's system to the class of bivariate distribution given by:

$$
H(x, y)=F(x) G(y)[1+\alpha A\{F(x)\} B\{G(y)\}]
$$

where $F(x)$ and $G(y)$ are two given univariate CDFs. $A\{F(x)\}$ and $B\{G(y)\}$ are two functions such that the limit of $A\{F(x)\}$ is 0 as $F(x)$ goes to 1 , and the limit 
of $B\{G(y)\}$ is 0 as $G(y)$ goes to 1 . This class of bivariate distributions would have $F(x)$ and $G(y)$ as its univariate marginals where again parameter $\alpha$ is a measure of dependence between $X$ and $Y$. If in Farlies Model, one takes $A\{F(x)\}=1-F(x)$, and $B\{G(y)\}=1-F(y)$, then this class of bivariate CDF reduces to Morgenstern's class of bivariate distributions. As long as $A\{F(x)\}$ and $B\{G(y)\}$ satisfy certain regularity, $H(x, y)$ could be a proper distribution function. Furthermore, this system contains the independence case when $\alpha=0$ as well.

\section{Plackett's Class of Bivariate Distributions}

Plackett(1965) introduced a one parameter class of bivariate distributions with given marginals. The method of construction is by the relationship:

$$
\psi=\frac{H(1-F-G+H)}{(F-H)(G-H)}
$$

Plackett showed that, for any fixed $\psi$ in the interval $(0, \infty)$, equation $(26)$ has a single root $H$ in the interval $H_{0}(x, y) \leq H(x, y) \leq H_{1}(x, y)$. Therefore, the function defined is a proper joint distribution function for some random variable $X$ and $Y$. According to Delores (1979), equation (26) is equivalent to a quadratic equation in $H$. That is,

$$
(\psi-1) H^{2}-[1+(F(x)+G(y))(\psi-1)] H+\psi F(x) G(y)=0
$$

In both methods, the resulting function $H(x, y)$ is a proper bivariate distribution function with marginals $F(x)$ and $G(y)$. In addition, this system of bivariate distribution is often referred to as contingency type distribution. 


\section{New Class of Bivariate Distribution Based on Weibull Mixture Distri- bution}

\section{Review of Univariate Mixture Weibull Distribution with BTFRF}

The Weibull distribution is one of the most commonly used distributions in modelling lifetime data, because of its flexibility. In the present study, Weibull distribution is used for modelling lifetime data both in the univariate case and in the bivariate case.

Definition A continuous random variable $X$ is said to have a Weibull distribution if its probability density function is given by:

$$
f(t)=\frac{\beta}{\eta}\left(\frac{t-\gamma}{\eta}\right)^{\beta-1} e^{-\left(\frac{t-\gamma}{\eta}\right)^{\beta}}
$$

where $\beta$ is a shape parameter, $\eta$ is a scale parameter, and $\gamma$ is a location parameter. In the present research we use the standard Weibull distribution where $\gamma=0$ and $\eta=1$. As a result, the cumulative distribution function is as below:

$$
F(t)=1-e^{-t^{\beta}}
$$

In the present case, the probability density function is reduced to:

$$
f(t)=\beta(t)^{\beta-1} e^{-(t)^{\beta}}
$$

and the corresponding failure rate function is given by:

$$
r(t)=\frac{f(t)}{S(t)}=\frac{\frac{\beta}{\eta}\left(\frac{t}{\eta}\right)^{\beta-1} e^{-\left(\frac{t}{\eta}\right)^{\beta}}}{e^{-\left(\frac{t}{\eta}\right)^{\beta}}}=\beta(t)^{\beta-1}
$$

Note that in (31), r(t) is increasing in $t$ if $\beta>1$, decreasing in $t$ if $\beta<1$, and it is a constant if $\beta=1$. Thus, a mixture Weibull distribution can be used to define a 
BTFRF survival function. Considering the case where the change points $a$ and $b$ are the same, that is, when lifetime only has a "burn-in" period and a "wear-out" period. If we can define the following failure rate function:

$$
r(t)= \begin{cases}\beta_{1} t^{\beta_{1}-1} & t \leq a \\ \beta_{2} t^{\beta_{2}-1} & t \geq a\end{cases}
$$

then the failure rate is bath-tub shaped if $\beta_{1}$ is less than 1 and $\beta_{2}$ is greater than 1 . In addition, since we want the function to be continuous at point $a$, the change point $a$ is decided by the relationship $\beta_{1} a^{\beta_{1}-1}=\beta_{2} a^{\beta_{2}-1}$.

\section{New Class of Bivariate Distribution with Mixture Weibull as Marginals}

From chapter 4, a bivariate distribution could be obtained if marginals $F(x)$ and $G(y)$ are given. Among three methods introduced, the first method, the Morgenstern(1956) class of bivariate distributions, was used in this research. Recall, if $F(x)$ and $G(y)$ are two arbitrary given distributions, then the Morgenstern class of bivariate distribution function, $H(x, y)$, which has the given marginals $F(x)$ and $G(y)$, is given by:

$$
H(x, y)=F(x) G(y)[1+\alpha(1-F(x))(1-G(y))]
$$

where parameter $\alpha$ is a measurement of dependence between $X$ and $Y$.

As a result, the survival function of $(X, Y)$ could be derived from the joint cumulative distribution function as follows:

$$
\begin{aligned}
\bar{H}(x, y) & =1+H(x, y)-H(x, \infty)-H(\infty, y) \\
& =1+H(x, y)-F(x)-G(y)
\end{aligned}
$$




$$
=1+F(x) G(y)[1+\alpha(1-F(x))(1-G(y))]-F(x)-G(y)
$$

Furthermore, using the survival function in (34), one can get the hazard gradients $r_{x}(x, y)$ and $r_{y}(x, y)$ respectively. The hazard gradients are given by:

$$
\begin{aligned}
r_{x}(x, y) & =\frac{-\frac{\partial}{\partial x} \bar{H}(x, y)}{\bar{H}(x, y)} \\
& =\frac{f(x) \bar{G}(y)[1-\alpha G(y)(\bar{F}(x)-F(x))]}{\bar{H}(x, y)}
\end{aligned}
$$

and

$$
\begin{aligned}
r_{y}(x, y) & =\frac{-\frac{\partial}{\partial y} \bar{H}(x, y)}{\bar{H}(x, y)} \\
& =\frac{g(y) \bar{F}(x)[1-\alpha F(x)(\bar{G}(y)-G(y))]}{\bar{H}(x, y)}
\end{aligned}
$$

If $r_{x}(x, y)$ and $r_{y}(x, y)$ are bath-tub shaped with respect to $x$ and $y$, respectively; then $H(x, y)$ will have a bivariate bath-tub shaped failure rate functions. In the present research, we consider the simplest case where $X$ and $Y$ are identically distributed. As a result, the change points for $X$ and for $Y$ are the same in this study, even though change points for $r_{x}(x, y)$ and $r_{y}(x, y)$ do not need to be identical in general. Let $X$ and $Y$ are identically distributed as a mixture of Weibull distributions with the common failure rate function:

$$
r(t)= \begin{cases}\beta_{1} t^{\beta_{1}-1} & t \leq a \\ \beta_{2} t^{\beta_{2}-1} & t \geq a\end{cases}
$$

where $\beta_{1}$ is less than 1 and $\beta_{2}$ is greater than 1 , which guaranteed that the failure rate functions for $X$ and $Y$ to be a U-shaped bath-tub shaped; respectively. Since $X$ 
and $Y$ are assumed to be identically distributed, we assume the change points to be the same. The value of the change points can be derived from the equation:

$$
\beta_{1} a^{\beta_{1}-1}=\beta_{2} a^{\beta_{2}-1}
$$

that is,

$$
a=\left(\frac{\beta_{1}}{\beta_{2}}\right)^{\frac{1}{\beta_{2}-\beta_{1}}}
$$

In addition, survival function could be obtained from the failure rate function according to the relationship of $S(t)=e^{-\int_{0}^{t} r(t) \mathrm{d} t}$. Therefore, the survival function is:

$$
S(t)= \begin{cases}e^{-t^{\beta_{1}}} & t \leq a \\ e^{-\left(a^{\beta_{1}}-a^{\beta_{2}}\right)-t^{\beta_{2}}} & t>a\end{cases}
$$

Furthermore, the marginal Cumulative Distribution Functions (CDFs) for $X$ and $Y$ are given by:

$$
F(x)= \begin{cases}1-e^{-x^{\beta_{1}}} & x \leq a \\ 1-e^{-\left(a^{\beta_{1}}-a^{\beta_{2}}\right)-x^{\alpha_{2}}} & x>a\end{cases}
$$

and

$$
G(y)= \begin{cases}1-e^{-y^{\beta_{1}}} & y \leq a \\ 1-e^{-\left(a^{\beta_{1}}-a^{\beta_{2}}\right)-y^{\alpha_{2}}} & y>a\end{cases}
$$

Using (41) and (42) for $F(x)$ and $G(y)$ in the joint CDF:

$$
H(x, y)=F(x) G(y)[1+\alpha(1-F(x))(1-G(y))]
$$


we obtain the following new class of bivariate lifetime distributions:

$$
H(x, y)= \begin{cases}\left(1-e^{-x^{\beta_{1}}}\right)\left(1-e^{-y^{\beta_{1}}}\right)\left[1+\alpha e^{-\left(x^{\beta_{1}}+y^{\beta_{1}}\right)}\right] & x \leq a, y \leq a \\ \left(1-e^{-x^{\beta_{1}}}\right)\left(1-c e^{-y^{\beta_{2}}}\right)\left[1+c \alpha e^{-\left(x^{\beta_{1}}+y^{\beta_{1}}\right)}\right] & x \leq a, y>a \\ \left(1-c e^{-x^{\beta_{2}}}\right)\left(1-e^{-y^{\beta_{1}}}\right)\left[1+c \alpha e^{-\left(x^{\beta_{2}}+y^{\beta_{1}}\right)}\right] & x>a, y \leq a \\ \left(1-c e^{-x^{\beta_{2}}}\right)\left(1-c e^{-y^{\beta_{1}}}\right)\left[1+c^{2} \alpha e^{-\left(x^{\beta_{2}}+y^{\beta_{2}}\right)}\right] & x>a, y>a\end{cases}
$$

where constant $c$ equals $e^{-\left(a^{\beta_{1}}-a^{\beta_{2}}\right)}, \beta_{1}$ is less than 1 , and $\beta_{2}$ is greater than 1 . Note that $H(x, y)$, the new class of bivariate lifetime distribution, has mixture Weibull distributions marginals. In the next section we investigate the bath-tub shaped failure rates property of this new class.

\section{Numerical and Graphical Investigation}

In order to investigate the bath-tub shaped failure rate property of the new class of distributions, some numerical and graphical analysis are needed. According to the definition from Chapter 3.2.1, $H(x, y)$ has BTFRFs when $r_{x}(x, y)$ is bath-tub shaped with respect to $x$ for all $y$, and $r_{y}(x, y)$ is bath-tub shaped with respect to $y$ for all $x$. Using equation (35), the hazard gradient $r_{x}(x, y)$ corresponding to $H(x, y)$ 
is derived as follows:

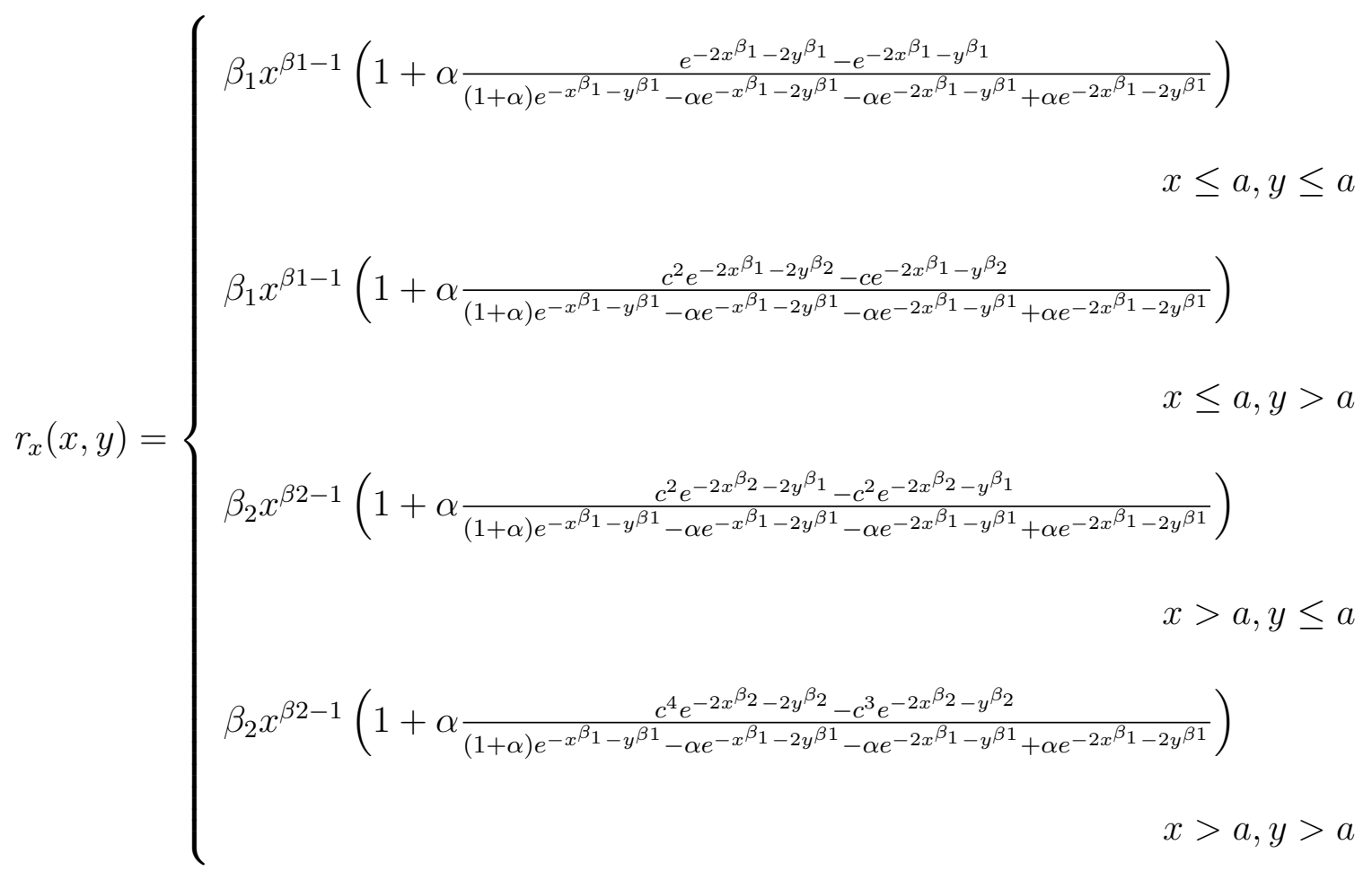

Since $X$ and $Y$ are identically distributed, $r_{y}(x, y)$ can be derived similarly. By changing the value of $\beta_{1}$ and $\beta_{2}$, different hazard gradients could be obtained. Since we are specifically interested in the shape, we use numerical and graphical methods to investigate this characteristic. First, a plot of $H(x, y)$ (equation (45)) reveals that this new bivariate distribution has the following form, for $\beta_{1}=0.3, \beta_{2}=1.5$, and $\alpha=0.5$. 


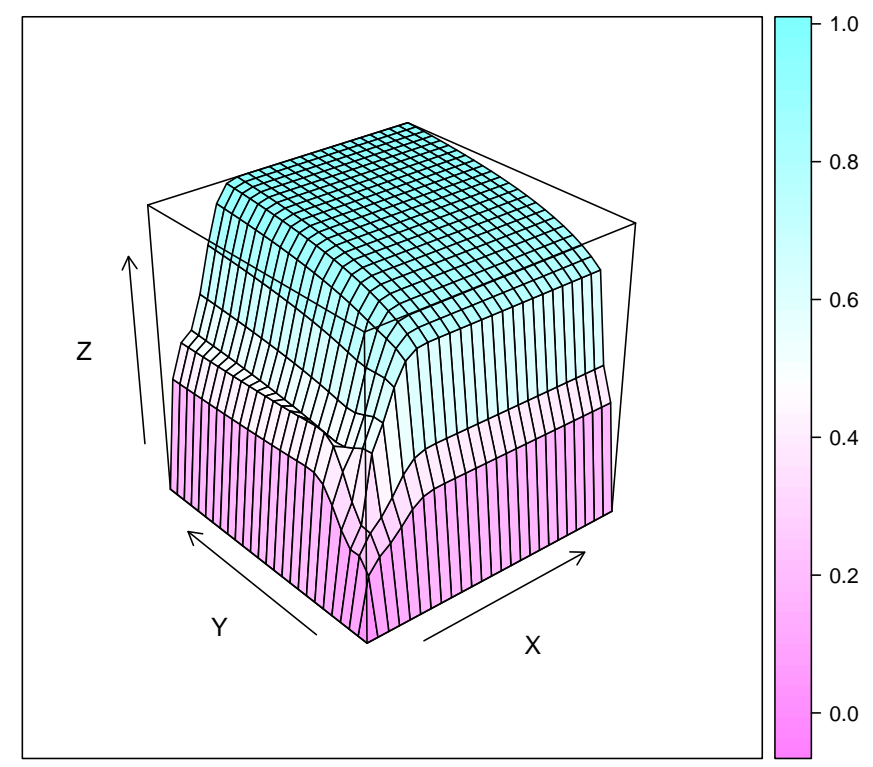

Figure 2: $H(x, y)$ when $\beta_{1}=0.3, \beta_{2}=1.5$, and $\alpha=0.5$

For this plot, $\alpha$ equals 0.5 , indicates that $X$ and $Y$ are positively associated; $\beta_{1}=$ $0.3<1$ and $\beta_{2}=1.5>1$, guarantee that the failure rates for the univariate marginals to be bath-tub shaped. From the plot, if $X \rightarrow \infty$ and $Y \rightarrow \infty, H(x, y)$ converges to 1. The plot of $H(x, y)$ gives some information about the shape of the distribution, but more importantly, it is of interest to analyse the plot of the hazard gradients $r_{x}(x, y)$ and $r_{y}(x, y)$. Since $X$ and $Y$ are identically distributed, only $r_{x}(x, y)$ is investigated; $r_{y}(x, y)$ would be symmetric. Two different cases are explored: the first case is when $X$ and $Y$ are independently distributed, and the second case is when $X$ and $Y$ are correlated. In the first case, $X$ and $Y$ are independently distributed with $\alpha=0$. The other requirements are $\beta_{1}<1$ and $\beta_{2}>1$. Therefore, for plot, we take $\beta_{1}=0.3$, and 
$\beta_{2}=1.5$. Other values can be chosen as well as long as $\beta_{1}<1$ and $\beta_{2}>1$. From values of $\beta_{1}$ and $\beta_{2}$, the value of change point, $a$, can be computed, which equals 0.26153. Part of the numerical results were summarized in Table 1 as shown below:

Table 1: Hazard Gradients Value

\begin{tabular}{ccccccccc}
\hline \hline$x$ & 0.19 & 0.20 & 0.21 & 0.22 & 0.23 & 0.24 & 0.25 & 0.26 \\
\hline$r_{x}(x, y)$ & 0.9594 & 0.9256 & 0.8945 & 0.8658 & 0.8393 & 0.8147 & 0.7917 & 0.7703 \\
\hline \hline$x$ & 0.27 & 0.28 & 0.29 & 0.30 & 0.31 & 0.32 & 0.33 & 0.34 \\
\hline$r_{x}(x, y)$ & 0.7794 & 0.7937 & 0.8078 & 0.8216 & 0.8352 & 0.8485 & 0.8617 & 0.8746 \\
\hline
\end{tabular}

where $x$ increases by 0.01 unit from 0.19 to 0.34 . Only $x$ and $r_{x}(x, y)$ are given in the table, since the change of $y$ does not affect the value of $r_{x}(x, y)$ if $x$ is given. Since $X$ and $Y$ are independent, the hazard gradient $r_{x}(x, y)$ is the same as the univariate failure rate function $r(x)$, which can be seen in the following:

$$
r_{x}(x, y)=\frac{-\frac{\partial}{\partial x} \bar{H}(x, y)}{\bar{H}(x, y)}=\frac{-\frac{\partial}{\partial x} \bar{F}(x) \bar{G}(y)}{\bar{F}(x) \bar{G}(y)}=\frac{-\frac{\partial}{\partial x} \bar{F}(x)}{\bar{F}(x)}=\frac{f(x)}{S(x)}=r(x)
$$

As Table 1 shows, $r_{x}(x, y)$ decreases as $x$ increases when $x$ is less than the change point $a=0.26153$, and $r_{x}(x, y)$ increases as $x$ increases for $x>a=0.26153$. Therefore, $r_{x}(x, y)$ is bath-tub shaped with respect to $x$ for all $y$. Likewise, the same procedure could verify that $r_{y}(x, y)$ is bath-tub shaped with respect to $y$ for all $x$. Hence, $H(x, y)$ is a bivariate lifetime distribution with BTFRFs based on the numerical results. Besides the numerical results, plot of $r_{x}(x, y)$ could also help us illustrate how $r_{y}(x, y)$ changes as $x$ and $y$ change. The plot of $r_{x}(x, y)$ is given below: 


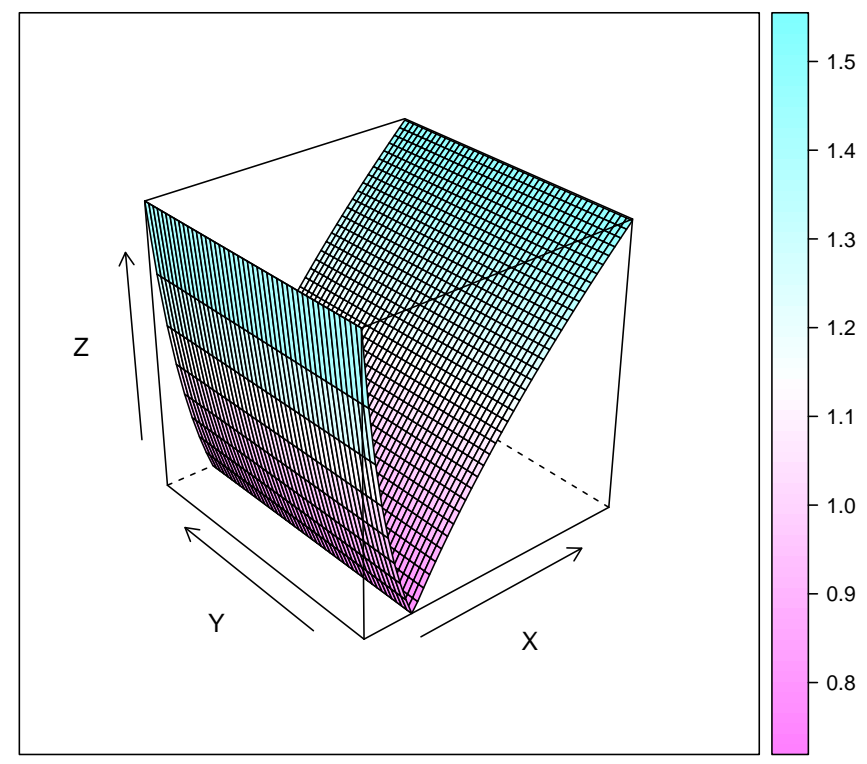

Figure 3: $r_{x}(x, y)$ when $\beta_{1}=0.3, \beta_{2}=1.5, \alpha=0$

From Figure 3, $r_{x}(x, y)$ decreases when $x$ is less than the change point $a$ and increases when $x$ is greater than $a$. The change of $y$ does not affect the value of $r_{x}(x, y)$ if $x$ is given because $\alpha=0$. Since this model does not have a constant failure rate period, one can regard this as a bath-tub shaped failure rate with only "burn-in" and "wearout" periods. For this case, $r_{y}(x, y)$ is exactly the same as $r_{x}(x, y)$, since $X$ and $Y$ are identically distributed. Figure 3 shows that $H(x, y)$ is a bivariate lifetime distribution with U-shaped or V-shaped BTFRFs.

Note that, two parameters $\beta_{1}$ and $\beta_{2}$ affect the shape of the hazard gradient. If the values of $\beta$ s are changed, then the rate at which the hazard gradient decreases during the "burn-in" period and the rate at which the hazard gradient increases 
during the "wear-out" period will be changed. In other words, different $\beta_{1}$ and $\beta_{2}$ could generate different plots with different slopes. For example, if one takes $\beta_{1}=0.5$ and $\beta_{2}=4$, a more "curved", more U-shaped, plot could be obtained:

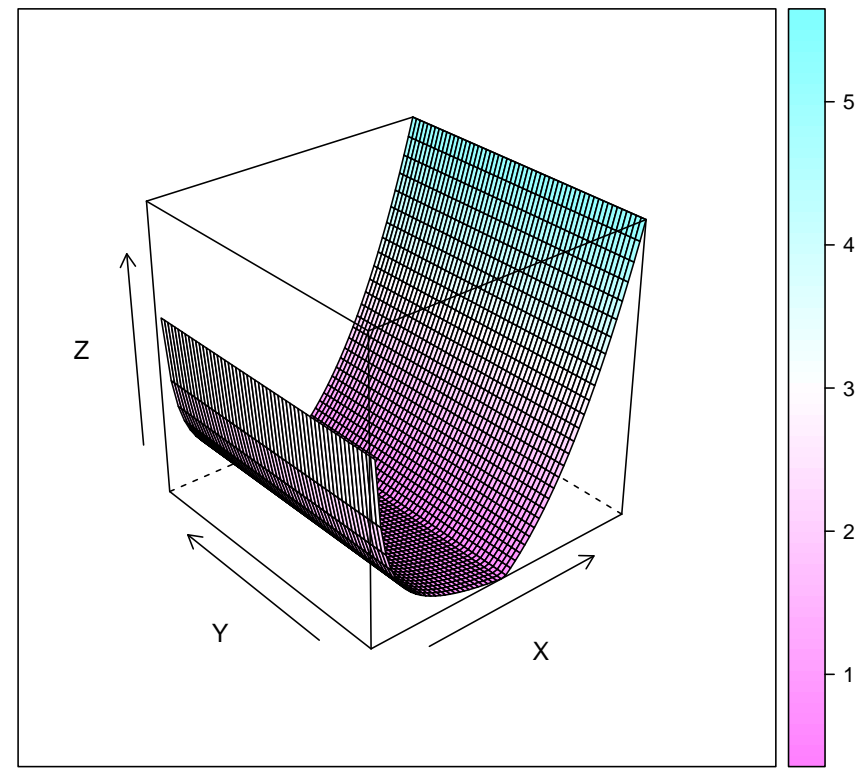

Figure 4: $r_{x}(x, y)$ when $\beta_{1}=0.5, \beta_{2}=4, \alpha=0$

In the example above, $X$ and $Y$ are still independent since $\alpha$ equals zero. However, the plot is more "curved" than the previous one because of different $\beta$ values. One thing remains the same is that the change of $y$ does not affect the shape of $r_{x}(x, y)$ because of the independence. However, the slope at which the hazard gradient decreases before the change point $a$ and the slope at which the hazard gradient increases after the change point $a$ have been changed. For the "burn in" period, the rate of decrease is more rapid at the beginning, but it gradually becomes less rapid as $x$ increases. For 
the "wear-out" period, the speed of the "wear-out" effect increases as time passes, whereas, in the previous plot, the speed of the "wear-out" effect seems to not change too much with the passage of time. In conclusion, the values of $\beta_{1}$ and $\beta_{2}$ are closely associated with the mortality rate at each time point, which is similar to the univariate situation where the $\beta$ values are closely associated with the failure rate. In conclusion, $\beta_{1}$ and $\beta_{2}$ parameters could affect the slope at which the failure rate decreases and the slope at which it increases; respectively.

Independence is a relatively strict condition, now we investigate the case where $X$ and $Y$ are not independent, which could be done by assigning a non-zero value between -1 and 1 to $\alpha$. Note that $\alpha$ is a measurement of association between $X$ and $Y$, but it is not the same as the correlation, which measures the strength of linear dependence between $X$ and $Y$. If $X$ and $Y$ are associated, the surface of the $r_{x}(x, y)$ and $r_{y}(x, y)$ are not as smooth as the previous case. That is, if $X$ and $Y$ are associated, $r_{x}(x, y)$ and $r_{y}(x, y)$ depend on values of $x$ and $y$ both. Figure 5 shows one example of the plot of $r_{x}(x, y)$ when $\alpha$ equals 0.5 ( $\beta$ s remain the same). 


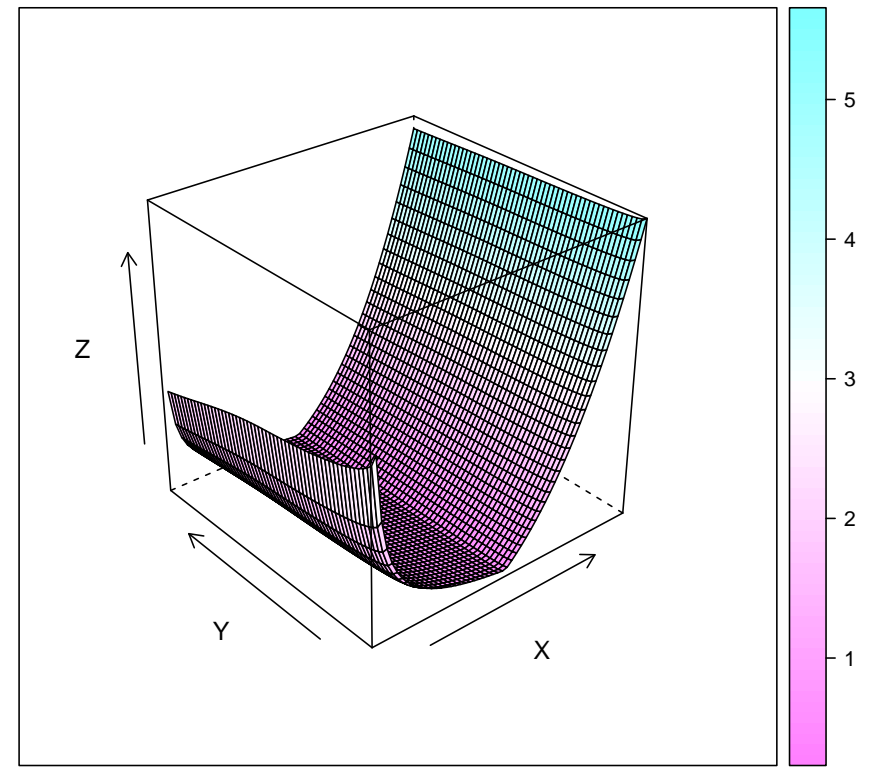

Figure 5: $r_{x}(x, y)$ when $\beta_{1}=0.5, \beta_{2}=4, \alpha=0.5$

Comparing Figure 5 and Figure 4, it is clear that the new value for the parameter $\alpha$ has changed the shape of $r_{x}(x, y)$, however, $r_{x}(x, y)$ is still bath-tub shaped with respect to $x$ for all $y$, and decreases with respect to $y$ for all $x$. The numerical values of $r_{x}(x, y)$ for some selected values of $x$ and $y$ are given in the following table. 
Table 2: $r_{x}(x, y)$ when $\alpha=0.5$

\begin{tabular}{|l|l|l|l|l|l|l|l|l|l|}
\hline \multicolumn{2}{|c|}{$r_{x}(x . y)$} & \multicolumn{10}{|c|}{$y$} \\
\cline { 2 - 9 } & 0.48 & 0.50 & 0.52 & 0.54 & 0.56 & 0.58 & 0.60 & 0.62 \\
\hline & 0.46 & 0.6540 & 0.6529 & 0.6519 & 0.6509 & 0.6500 & 0.6489 & 0.6478 & 0.6466 \\
\hline 0.48 & 0.6415 & 0.6405 & 0.6395 & 0.6386 & 0.6377 & 0.6367 & 0.6356 & 0.6345 \\
\hline 0.50 & 0.6298 & 0.6288 & 0.6279 & 0.6270 & 0.6261 & 0.6251 & 0.6241 & 0.6230 \\
\hline 0.52 & 0.6187 & 0.6178 & 0.6169 & 0.6160 & 0.6151 & 0.6142 & 0.6132 & 0.6122 \\
\hline \multirow{4}{*}{$x$} & 0.54 & 0.6082 & 0.6073 & 0.6065 & 0.6056 & 0.6048 & 0.6039 & 0.6030 & 0.6019 \\
\hline 0.56 & 0.6291 & 0.6282 & 0.6273 & 0.6264 & 0.6256 & 0.6247 & 0.6237 & 0.6227 \\
\hline & 0.58 & 0.7002 & 0.6992 & 0.6983 & 0.6973 & 0.6964 & 0.6955 & 0.6944 & 0.6933 \\
\hline & 0.60 & 0.7768 & 0.7757 & 0.7747 & 0.7736 & 0.7727 & 0.7716 & 0.7705 & 0.7692 \\
\hline & 0.62 & 0.8590 & 0.8578 & 0.8567 & 0.8556 & 0.8545 & 0.8534 & 0.8522 & 0.8508 \\
\hline & 0.64 & 0.9471 & 0.9458 & 0.9446 & 0.9434 & 0.9423 & 0.9411 & 0.9397 & 0.9383 \\
\hline
\end{tabular}

Table 2 clearly shows that, at any fixed value of $y, r_{x}(x, y)$ is monotonically decreasing with respect to $x$ for $x \leq a$, where the value of change point $a$, when $\beta_{1}=0.5, \beta_{2}=4$, and $\alpha=0.5$, is given by:

$$
a=\left(\frac{\beta_{1}}{\beta_{2}}\right)^{\frac{1}{\beta_{2}-\beta_{1}}}=\left(\frac{0.5}{4}\right)^{\frac{1}{4-0.5}}=0.55204
$$

Also, note that, $r_{x}(x, y)$ is a decreasing function of $x$ for $x \leq a$ and is a increasing function of $x$ when $x>a$ at each $y$. Hence, when $\alpha=0.5$, again $H(x, y)$ is a BTFRF distribution. Similar result also holds for $r_{y}(x, y)$ since $X$ and $Y$ are identically distributed. That is, $r_{y}(x, y)$ is bath-tub shaped with respect to $y$ at each $x$. Therefore, the bivariate CDF, $H(x, y)$, is a new class of BTFRF distributions.

Note that for the "burn-in" period, $r_{x}(x, y)$ is decreasing with respect to both $x$ and $y$. But, the rate at which $r_{x}(x, y)$ decreases with respect to $y$ is faster than 
the rate at which $r_{x}(x, y)$ decreases with respect to $x$. That is, for a fixed $y, r_{x}\left(x_{1}, y\right)$ is greater than $r_{x}\left(x_{2}, y\right)$, if $x_{1}$ is less than $x_{2}$ during the "burn-in" period. However, $r_{x}\left(x_{1}, y_{1}\right)$ could be less than $r_{x}\left(x_{2}, y_{2}\right)$, if $y_{1}$ is significantly greater than $y_{2}$. Our numerical simulations exhibit similar patterns as long as $\beta_{1}<1$ and $\beta_{2}>1$, regardless of the value of $\alpha$. That is, the general shape of failure rates are (U-Shaped) BTFRF, but the slopes of curvatures are different.

This new class of bivariate lifetime distributions with BTFRFs developed here is derived from the utilization of a bivariate class of CDF given by Morgenstern. It will be of interest to define and investigate other classes of multivariate BTFRF distributions based on other multivariate extensions. This work provides a basis for many other possible research in this concept. 


\section{Possible Future Work}

One important future line of research is to extend the results obtained here to a more general case where the failure rate has a constant period after the "burn-in" period. That is, the change points $a$ and $b$ in the model are not equal. Since in this case, the hazard gradient has three periods; "burn-in", constant, and "wear-out" periods. Thus the joint cumulative distribution function will have nine regions as given by (20) instead of the four regions for the case that we discussed in the previous section. Obviously, the problem will become more complicated.

Another generalization for the future work is to investigate the characteristics of this new class of bivariate lifetime distributions when the marginals $X$ and $Y$ are not identically distributed. The procedures will be the same except the computations will be more extensive. In the example that is illustrated, $X$ and $Y$ are set to be identically distributed in order to simplify the computation. However, $X$ and $Y$ could have different distributions in a more general situation. For example, $X$ could be a mixture Weibull distribution that has a BTFRF, and $Y$ could be another existing distribution that has a BTFRF. The question now is that: "Does this class of bivariate lifetime distribution still have bath-tub shaped failure rate functions?"

The third area for the future research is the investigation of statistical properties for the new model, which includes the estimation of the parameters of the new model, confidence intervals, testing hypothesis about the parameters, including the change points and bivariate BTFRF goodness-of-fit for bivariate failure times. 


\section{REFERENCES}

B. L. Amstadter. (1977). Reliability Mathematics: Fundamentals, Practices, Procedures McGraw-Hill.

Nataf, M.A. (1962). Determination des distributions de probabilites dont les marges sont donnes, Comptes Renud de lAcademie des. Sciences, 225: 42-43.

R.E. Barlow and F. Proschan. (1975). Statistical Theory of Reliability and Life Testing Probability Models, p. 55.

Morgenstern, D. (1956). Einfache Beispiele zweidimensionaler Verteilungen. Mitteilungsblatt fur Mathematische Statistik, 8: 234-235.

Ebeling, C.E. (1997). An Introduction to Reliability and Maintainability Engineering.

Barlow, R.E., Proschan, F. (1975). Statistical Theory of Reliability and Life Testing. Holt, Rinehart and Winston, New York.

Ghitany, M.E. (2004). The monotonicity of the reliability measures of the beta distribution. Applied Mathematics Letters, 17: 1277-1283.

Ronald E. Glaser. (1980). Bathtub and Related Failure Rate Characterizations. Journal of the American Statistical Association, 75: 667-672.

Farlie,D.J.G. (1960). The Performance of Some Correlation Coefficients for a General Bivariate Distribution. Biometrika, 47: 307-323.

E. Halley. (1693). An estimate of the degrees of the mortality of mankind, drawn from curious tables of the births and funerals at the city of Breslau; with an attempt to ascertain the price of annuities upon lives. Philosophical Trans. Royal Society of London, 17: 596-610.

B. F. Jensen. (1989). Component failures based on flaw distributions. Proc. Annu. Reliability and Maintainability Symp., p. 9195

Johnson, N.L. and Kotz, S. (1975). A Vector of Multivariate Hazard Rate. J. Multivariate Anal, 5: 53-66.

Albert W. Marshall (1975). Some Comments on the Hazard Gradient. Stochastic Processes and their Applications 3: 293-300. 
Xie, M. and Lai, C.D. (1995). Reliability Analysis Using an Additive Weibull Model with bathtub-shaped failure rate function. Reliability Engineering and System Safety 52: 87-93.

Basu, A.P. (1971). Bivariate Failure Rate. American Statistical Association, 66: 103-104.

R.L. Plackett. (1965). A Class of Bivariate Distributions. American Statistical Association, 61: 516-22.

Mudholkar, G.S., Srivastava, D.K., and Freimer, M. (1995). The exponentiated Weibull family. Technometrics 37: 436-445.

Mudholkar, G.S., Srivastava, D.K., Kollia, G.D. (1996). A generalization of the Weibull distribution with application to the analysis of survival data. Journal of the American Statistical Association, 91: 1575-1583.

Marshall, A.W. (1975). Some comments on the hazard gradient. Stochastic Process. Appl, 3: 293-300. 